\title{
Bounding Skeletons, Locally Scoped Terms and Exact Bounds for Linear Head Reduction
}

\author{
Pierre Clairambault \\ Computer Laboratory, University of Cambridge \\ pierre.clairambault@cl.cam.ac.uk
}

\begin{abstract}
Bounding skeletons were recently introduced as a tool to study the length of interactions in Hyland/Ong game semantics. In this paper, we investigate the precise connection between them and execution of typed $\lambda$-terms. Our analysis sheds light on a new condition on $\lambda$-terms, called local scope. We show that the reduction of locally scoped terms matches closely that of bounding skeletons. Exploiting this connection, we give upper bound to the length of linear head reduction for simply-typed locally scoped terms. General terms lose this connection to bounding skeletons. To compensate for that, we show that $\lambda$-lifting allows us to transform any $\lambda$-term into a locally scoped one. We deduce from that an upper bound to the length of linear head reduction for arbitrary simply-typed $\lambda$-terms. In both cases, we prove the asymptotical optimality of the upper bounds by providing matching lower bounds.
\end{abstract}

\section{Introduction}

In the last two decades there has been a growing interest in the study of quantitative or intensional aspects of higher-order programs; in particular, the study of their complexity has generated a lot of effort. In the context of the $\lambda$-calculus, the first result that comes to mind is the work by Schwichtenberg [14], later improved by Beckmann [2], establishing upper bound to the length of $\beta$-reduction sequences for simply-typed $\lambda$-calculus. In the somewhat related line of work of implicit complexity, type systems have been developed to characterize extensionally certain classes of functions, such as polynomial [10] or elementary [8] time. Such systems rely on a soundness theorem establishing that well-typed terms normalize in a certain restricted time, which is itself established using syntactic methods that are specific to the system being studied. This calls for the development of syntax-independent tools to study precisely the execution time of higher-order programs. The present paper is a step towards that goal.

In [4], in the context of Hyland-Ong game semantics, we showed that provided some size information on strategies we could give a bound to the length of their interactions. This was done by annotating each step of an interaction sequence with a finite tree of natural numbers - hereby called a bounding skeleton ${ }^{1}$ - and showing that progress in the interaction amounts to a simple reduction on

\footnotetext{
1 They were called agents in [4].
} 
the bounding skeleton. We gave bounds to the length of this reduction, hence bounding with it the length of the interaction sequence. The strength of this approach is that the games model is syntax-independent: in the variant considered in [4], it accommodates the simply-typed $\lambda$-calculus possibly with computational effects such as non-determinism, control, or ground type references. Its key weakness however, is that the direct connection between game-theoretic interaction and execution has only been made explicit [6] for pure simply-typed $\lambda$-terms of the form $M N_{1} \ldots N_{p}$, where $M$ and the $N_{i}$ s are $\eta$-long Böhm trees - we will call such terms game situations. Although terms can be transformed into game situations (as briefly described in [4]), the transformation is very inefficient and yields bounds that are sub-optimal and not very informative. For bounding skeletons to be a useful tool in complexity analysis, it is crucial to relate them directly to the execution of programs, without the detour by game semantics. Such a connection is non-trivial, as the dynamics of reduction in all generality is much more complicated than for game situations.

In this paper, we develop such a connection. This is done by introducing a new structural condition on terms, local scope, that ensures that information only flows locally through redexes, and not remotely through variables shared by distant subterms. We show that the reduction of $\eta$-long, locally scoped terms can be directly simulated within bounding skeletons. Using this property and (a small optimization of) our results in [4] on bounding skeletons, we deduce exact bounds to the execution time of locally scoped terms. We also show that the operation of $\lambda$-lifting [12] transforms arbitrary terms into locally scoped ones, and exploit this transformation to give exact bounds for the execution time of arbitrary simply-typed $\lambda$-terms.

Related works. There are multiple approaches to the complexity analysis of higher-order programs, but they seem to separate into two major families. On the one hand, Beckmann [2], extending earlier work by Schwichtenberg [14], gave exact bounds to the maximal length of $\beta$-reduction on simply-typed $\lambda$ terms. His analysis uses very basic information on the terms (their length, or height, and order), but gives bounds that are in general very rough. On the other hand other groups, including Dal Lago and Laurent [13], De Carvalho [9], or Bernardet and Lengrand [3], use semantic structures (respectively, game semantics, relational semantics, or non-idempotent intersection types) to capture abstractly the precise complexity of particular terms. Their bounds are much more precise on particular terms, but require information on the terms whose extraction is in general as long to obtain as actual execution. The present work belongs to the first family. However, unlike Beckmann and Schwichtenberg the reduction we consider is linear head reduction, which is the notion of execution implemented by abstract machines [7] and is therefore much closer to the actual execution of functional programming languages.

Outline. In Section 2, we start by introducing linear head reduction along with bounding skeletons, and recall the main result of [4]. In Section 3, we introduce local scope, show our simulation result and deduce exact bounds on the length of linear head reduction on locally scoped terms. Finally in Section 4, we show 
how to use $\lambda$-lifting to transform arbitrary terms into locally scoped ones, and deduce exact bounds for linear head reduction on general terms.

\section{Preliminaries}

In this section, we start by recalling some of the background of this research. The natural starting point is linear head reduction [7], which can be seen as a direct implementation on $\lambda$-terms of the notion of execution performed by abstract machines. We will then turn to the presentation of bounding skeletons: we will recall the results of [4] on the length of their reductions, along with a small improvement.

\subsection{Linear head reduction}

We work here with the simply-typed $\lambda$-calculus à la Church, i.e. the variables are explicitly annotated with types (although we often omit the annotations for the sake of readability). Types are built from a unique atom $o$ and the arrow constructor $\rightarrow$. We suppose that for every type $A$, there is a constant $*_{A}: A$ of type $A$. We will often omit the index and write $*$. As usual, we write $\mathrm{fv}(M)$ for the set of free variables of a term $M$. The typing relation $\Gamma \vdash M: A$ is defined by the usual deduction rules for simply-typed $\lambda$-calculus. All the terms considered in this paper are supposed well-typed. Note that our choices - only one atom, each type is inhabited - merely make the presentation simpler and are not strictly required for our results to hold.

This work focuses strongly on linear substitution, for which only one variable occurrence is substituted at a time. In this situation, it is convenient to have a distinguished notation for particular occurrences of variables. We will use the notations $x_{0}, x_{1}, \ldots$ to denote particular occurrences of the same variable $x$ in a term $M$. When in need of additional variable identifiers, we will use $x^{1}, x^{2}, \ldots$. Sometimes, we will still denote occurrences of $x$ by just $x$ when their index is not relevant. Although it is not the focus of this development, we will occasionally also refer to $\beta$-reduction: it is the standard rewriting rule on $\lambda$-terms, defined by $(\lambda x . M) N \rightarrow_{\beta} M[N / x]$, where $M[N / x]$ is the substitution of all occurrences of the variable $x$ by $N$, applied in any position within $M$. We write $\equiv_{\beta}$ for the corresponding equivalence relation. If $x_{0}$ is a specific occurrence of $x$, we will use $M\left[N / x_{0}\right]$ for the substitution of $x_{0}$ by $N$, leaving all other occurrences of $x$ unchanged. We assume Barendregt's convention and consider all terms up to $\alpha$-equivalence (so, substitution involves renaming of bound variables).

Intuitively, linear head reduction proceeds as follows. We first locate the head variable occurrence, i.e. the leftmost variable occurrence in the term $M$. Then we locate the abstraction, if any, that binds this variable. Then we locate (again if it exists) the subterm $N$ of $M$ in argument position for that abstraction, and we substitute the head occurrence by $N$. We touch neither the other occurrences of $x$ nor the redex. It is worth noting that locating the argument subterm can be delicate, as it is not necessarily part of a $\beta$-redex. For instance 
in $\left(\lambda y^{A} \cdot\left(\lambda x^{B} \cdot x_{0} M\right)\right) N_{1} N_{2}$, we want to replace $x_{0}$ by $N_{2}$, even though $N_{2}$ is not directly applied to $\lambda x^{B} \cdot x_{0} M$. Therefore, the notion of redex will be generalized.

Note that a term is necessarily of the form $* M_{1} \ldots M_{n}, x_{0} M_{1} \ldots M_{n}, \lambda x . M$ or $(\lambda x . M) M_{1} \ldots M_{n}$. That will be used quite extensively to define and reason on linear head reduction. The length of a term $M$ is the number of characters in $M$, i.e. $\mathrm{l}(*)=1, \mathrm{l}\left(x_{0}\right)=1, \mathrm{l}(\lambda x . M)=\mathrm{l}(M)+1, \mathrm{l}\left(M_{1} M_{2}\right)=\mathrm{l}\left(M_{1}\right)+$ $\mathrm{l}\left(M_{2}\right)$. Its height is $\mathrm{h}(*)=0, \mathrm{~h}\left(x_{0}\right)=1, \mathrm{~h}(\lambda x \cdot M)=\mathrm{h}(M), \mathrm{h}\left(M_{1} M_{2}\right)=$ $\max \left(\mathrm{h}\left(M_{1}\right), \mathrm{h}\left(M_{2}\right)+1\right)$.

Definition 1. Given a term $M$, we define its set of prime redexes. They are written as pairs $(\lambda x, N)$ where $N$ is a subterm of $M$, and $\lambda x$ is used to denote the (if it exists, necessarily unique by Barendregt's convention) subterm of $M$ of the form $\lambda x . N^{\prime}$. We define the prime redexes of $M$ by induction on its length, distinguishing several cases depending on the form of $M$.

- If $M=* M_{1} \ldots M_{n}$, then $M$ has no prime redex.

- If $M=x_{0} M_{1} \ldots M_{n}$, then $M$ has no prime redex.

- If $M=\lambda x \cdot M^{\prime}$, then $M$ has the prime redexes of $M^{\prime}$.

- If $M=\left(\lambda x . M^{\prime}\right) M_{1} \ldots M_{n}$, then the prime redexes of $M$ are $\left(\lambda x, M_{1}\right)$ plus those of $M^{\prime} M_{2} \ldots M_{n}$.

The head occurrence of a term $M$ is the leftmost occurrence of a variable or constant in $M$. If $(\lambda x, N)$ is a prime redex of $M$ where the head occurrence of $M$ is an occurrence $x_{0}$ of the variable $x$, then the linear head reduct of $M$ is $M^{\prime}=M\left[N / x_{0}\right]$. We write $M \rightarrow_{\mathrm{lhr}} M^{\prime}$.

Example 1. As an example, we give the linear head reduction sequence of the term $(\lambda f . \lambda x . f(f x))(\lambda y \cdot y)$.

$$
\begin{aligned}
(\lambda f . \lambda x . f(f x))(\lambda y . y) & \rightarrow_{\mathrm{lhr}}(\lambda f \cdot \lambda x \cdot(\lambda z \cdot z)(f x))(\lambda y \cdot y) \\
& \rightarrow_{\mathrm{lhr}}(\lambda f \cdot \lambda x \cdot(\lambda z \cdot f x)(f x))(\lambda y \cdot y) \\
& \rightarrow_{\mathrm{lhr}}(\lambda f \cdot \lambda x \cdot(\lambda z \cdot(\lambda u \cdot u) x)(f x))(\lambda y \cdot y) \\
& \rightarrow_{\mathrm{lhr}}(\lambda f \cdot \lambda x \cdot(\lambda z \cdot(\lambda u . x) x)(f x))(\lambda y \cdot y)
\end{aligned}
$$

At this point the reduction stops since the head occurrence is an occurrence of $x$, and the corresponding abstraction subterm is not part of a prime redex.

We will abbreviate linear head reduction by lhr. It is straightforward to see that lhr is compatible with $\beta$-reduction, in the sense that if $M \rightarrow_{\mathrm{lhr}} M^{\prime}$ we have $M \equiv{ }_{\beta} M^{\prime}$. Just as for $\beta$-reduction, lhr always terminates on well-typed terms, let us denote by $\mathcal{N}(M)$ the length of the reduction sequence of $M$. Since redexes for lhr are not necessarily $\beta$-redexes, it will be necessary to consider the following generalization of redexes:

Definition 2 (Generalized redex). The generalized redexes of a term $M$ are the prime redexes of all subterms of $M$. In particular, all prime redexes are generalized redexes. 
Example 2. Consider the following $\lambda$-term:

$$
M=(\lambda x \cdot x)((\lambda y \cdot(\lambda z \cdot u)) v w)
$$

The only prime redex of $M$ is $(\lambda x,(\lambda y \cdot(\lambda z \cdot u)) v w)$, and it is therefore also a generalized redex. The two other generalized redexes are $(\lambda y, v)$, which is also a $\beta$-redex, and $(\lambda z, w)$, which is not.

\subsection{Bounding skeletons}

This section focuses on a pivotal notion of this paper, that of a bounding skeleton. Intuitively, it is what is left of a term when all precise dynamic information is forgotten, and only the structural size information necessary to study termination is retained. Formally, a bounding skeleton is a finite tree whose nodes and edges are labeled by natural numbers. We write:

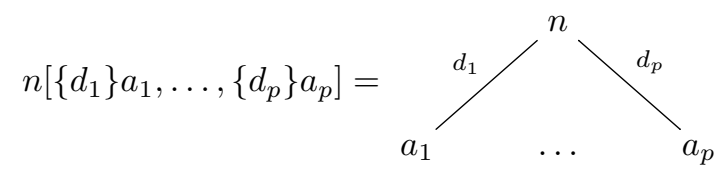

This notion was introduced in [4] where it was extracted from game semantics, and more precisely from the notion of pointing sequence central to Hyland-Ong games [11], but also appearing crucially in the earlier work of Coquand [5]. Bounding skeletons arise as measures of positions in pointing sequences, progress in the sequence corresponding to reduction of the skeleton. By the operational content of game semantics [6], bounding skeletons can also be seen as measures of terms obtained by lhr from a term of a particular form called a game situation. A game situation is a term of the form $M N_{1} \ldots N_{n}$, where $M: A_{1} \rightarrow \ldots \rightarrow$ $A_{n} \rightarrow o$ and $N_{i}: A_{i}$ are closed $\eta$-long Böhm trees - the terminology is motivated by the strong geometric correspondence between $\eta$-long Böhm trees and the innocent strategies of [11]. We know by the result of Danos, Herbelin and Regnier [6] that the lhr sequence of $M N_{1} \ldots N_{n}$ is in step-by-step correspondence with the game-theoretic interaction between the corresponding strategies $\llbracket M \rrbracket$ and $\llbracket N_{i} \rrbracket$. To illustrate how bounding skeletons arise from lhr of game situations, let us suppose for simplicity that $M:(A \rightarrow o) \rightarrow o$ and that $M$ has the form $\lambda x . x M^{\prime}$ with $M^{\prime} \eta$-long Böhm tree of type $A$, possibly including $x$ as a free variable. Then we have the lhr step:

$$
\left(\lambda x . x M^{\prime}\right) N \rightarrow_{\mathrm{lhr}}\left(\lambda x . N M^{\prime}\right) N
$$

Therefore, a situation with a closed Böhm tree $M$ applied to a closed Böhm tree $N$ is reduced to a closed Böhm tree $N$ applied to an open Böhm tree $M^{\prime}$, along with an environment associating $x$ to the closed Böhm tree $N$. In other words:

$$
M \star N \rightarrow N \star M^{\prime\{x \mapsto N\}}
$$


where the notation used will remain informal, but should be clear nonetheless. This can be represented by the following operation on trees of terms:

$$
\begin{array}{ccc} 
& & N \\
M & & \mid \\
! & \rightarrow & M^{\prime} \\
N & & \mid \\
& & N
\end{array}
$$

Replacing the terms by some measure of size (which will be made precise later in the paper) and annotating the edges with a measure of their types, these trees give rise to bounding skeletons, and this reduction appears as an instance of the following non-deterministic rule, illustrated in Figure 1.

$$
n\left[\left\{d_{1}\right\} a_{1}, \ldots,\left\{d_{p}\right\} a_{p}\right] \rightarrow_{\mathrm{bs}} a_{i} \cdot d_{i}-1(n-1)\left[\left\{d_{1}\right\} a_{1}, \ldots,\left\{d_{p}\right\} a_{p}\right]
$$

where $n, d_{i} \geq 1$ and $a \cdot{ }_{d} b$ denotes the skeleton $n\left[\left\{d_{1}\right\} a_{1}, \ldots,\left\{d_{p}\right\} a_{q},\{d\} b\right]$, for two skeletons $a=n\left[\left\{d_{1}\right\} a_{1}, \ldots,\left\{d_{p}\right\} a_{q}\right]$ and $b$.

This observation still applies with further reductions of $M N$, as we established in [4] through game semantics. Along with bounds to the length of reduction of bounding skeletons, this allowed us to bound the maximum length of $\mathrm{lhr}$ sequences starting from game situations. We also gave a bound for regular terms, relying on a very rough translation of arbitrary terms into game situations - as a result, this bound was far from optimal and not very informative. We aim in this paper to study the direct connection of bounding skeletons and syntax outside of game situations and independently on game semantics.
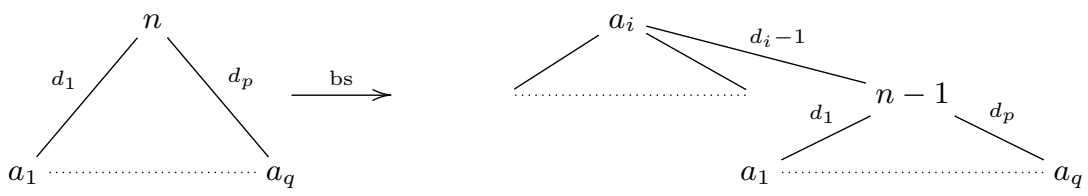

Fig. 1. Rewriting rule on skeletons

Remark 1. Note that reduction is set to only happen in root position, i.e. at the root of the tree. Generalizing it to apply deeper leads to pathological behavior. For instance deep reduction does not terminate on the variant without edge labels, whereas the standard (root) reduction does. It is not known whether deep reduction terminates in the presence of edge labels, or to which extent the relationship with syntactic reduction is preserved - the correspondence with game semantics is lost.

The main result of [4] is a bound on the length of reduction for bounding skeletons. The bound we state here is in fact a minor improvement of the result 
in [4], however the tools and methods to get it are the same. Therefore to save space, we omit the details of the optimization. As for terms, we write $\mathcal{N}(a)$ for the norm of a bounding skeleton $a$, i.e. the length of its longest reduction sequence. We also write $\max (a)$ for the highest node label in $a$, ord $(a)$ for the order of $a$, i.e. the highest edge label in $a$ and $\operatorname{depth}(a)$ for the depth of $a$, i.e. the maximal depth of a node in $a$, the root being at depth 1 . Here, log denotes the logarithm to base 2 and the tower of exponentials $2_{n}^{p}$ is defined by $2_{0}^{p}=p$ and $2_{n+1}^{p}=2^{2^{p}}$.

Theorem 1 (Upper bound). If $\operatorname{ord}(a), \operatorname{depth}(a), \max (a) \geq 1$, then

$$
\mathcal{N}(a) \leq 2_{\operatorname{ord}(a)-1}^{\operatorname{depth}(a) \log (\max (a)+1)}
$$

Constructions. In defining the interpretation of terms as bounding skeletons, we will make use of the following constructions. If $\left(a_{i}\right)_{1 \leq i \leq n}$ is a finite family of bounding skeletons, then writing $a_{i}=n_{i}\left[\left\{d_{i, 1}\right\} b_{i, 1}, \ldots,\left\{d_{i, p_{i}}\right\} b_{i, p_{i}}\right]$, we define:

$$
\begin{aligned}
& \bigsqcup_{i=1}^{n} a_{i}=\left(\max _{1 \leq i \leq n} n_{i}\right) \cdot\left[\left\{d_{i, j}\right\} b_{i, j} \mid 1 \leq i \leq n \& 1 \leq j \leq p_{i}\right] \\
& \sum_{i=1}^{n} a_{i}=\left(\sum_{i=1}^{n} n_{i}\right) \cdot\left[\left\{d_{i, j}\right\} b_{i, j} \mid 1 \leq i \leq n \& 1 \leq j \leq p_{i}\right]
\end{aligned}
$$

so, they either take the maximum or the sum of the roots, and simply append all the subtrees of the $a^{i}$ s. In the binary case, we write as usual + for the sum. Finally, each natural number $n$ can be seen as an atomic bounding skeleton $n$ [] without subtrees, still denoted by $n$. That should never cause any confusion.

Embedding. The norm of a bounding skeleton is unchanged by permutation of subtrees, or merging of identical subtrees, and is only increased by an increase of labels. If $a=n\left[\left\{d_{1}\right\} a_{1}, \ldots,\left\{d_{p}\right\} a_{p}\right]$ and $a^{\prime}=n^{\prime}\left[\left\{d_{1}^{\prime}\right\} a_{1}^{\prime}, \ldots,\left\{d_{p^{\prime}}^{\prime}\right\} a_{p^{\prime}}^{\prime}\right]$, we say that $a$ embeds in $a^{\prime}$, written $a \hookrightarrow a^{\prime}$, if $n \leq n^{\prime}$ and for any $i \in\{1, \ldots, p\}$ there exists $j \in\left\{1, \ldots, p^{\prime}\right\}$ such that $d_{i} \leq d_{j}^{\prime}$ and $a_{i} \hookrightarrow a_{j}^{\prime}$. Then we have:

Lemma 1 (Embedding lemma). If $a \hookrightarrow b$, then $\mathcal{N}(a) \leq \mathcal{N}(b)$.

We illustrate the reduction in Figure 2, where at each step we emphasize the subtree selected non-deterministically for the next reduction step. For conciseness, we also do not represent the subtrees under a node labeled 0 , as they can play no further part in the reduction.

\section{Locally scoped terms and bounding skeletons}

This section explains the direct connection between linear head reduction and the reduction of bounding skeletons. We will first introduce locally scoped terms, for which this connection holds, then prove their simulation within bounding skeletons, and finally deduce bounds for the length of their reduction. 


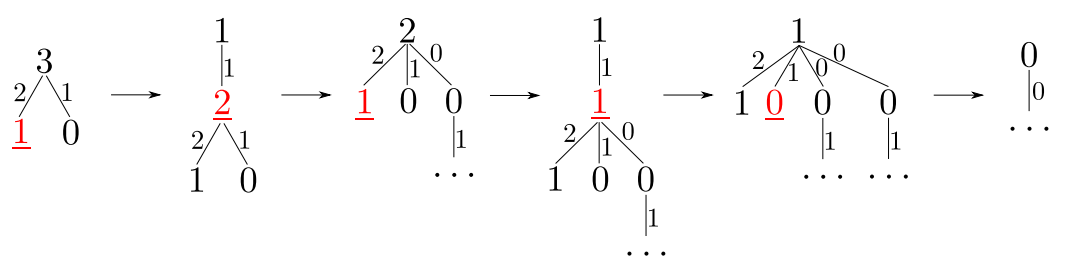

Fig. 2. Example reduction sequence on bounding skeletons

\subsection{Locally scoped terms}

Define inductively a closure as an open term $M$ along with an environment $\sigma$, mapping free variables of $M$ to closures of the same type. We say that a closure $M^{\sigma}$ is hereditarily normal when $M$ is $\beta$-normal and $\eta$-long, and when for any $x \in \mathrm{fv}(M)$, the closure $\sigma(x)$ is hereditarily normal. Hereditarily normal closures are very close to bounding skeletons: from a hereditarily normal closure $M^{\sigma}$ one can obtain a bounding skeleton having the height of $M$ as root, and the bounding skeletons corresponding to $\sigma(x)$ for $x \in \mathrm{fv}(x)$ as subtrees.

Although we will not make this formal in this paper, our simulation of lhr in bounding skeletons exploits that some terms can be represented as hereditarily normal closures. For instance, take the term:

$$
K_{1}=\left(\lambda x^{o \rightarrow o} \cdot\left(\lambda y^{o \rightarrow o} . y\right) x\right)\left(\lambda z^{o} . z\right)
$$

The term $K_{1}$ is faithfully represented by the hereditarily normal closure:

$$
y^{y \mapsto x^{x \mapsto \lambda z . z}}
$$

From that, we see that $K_{1}$ corresponds (ignoring edge labels) to the bounding skeleton 1[1[1]]. Note in passing that $K_{1}$ reduces to $(\lambda x .(\lambda y . x) x)(\lambda z . z)$, which by the same idea as above corresponds to the hereditarily normal form $x^{x \mapsto \lambda z . z}$, and to the bounding skeleton $1[1]$ - which embeds in the bs-reduct $1[1,0[1[1]]]$ of $1[1[1]]$, so the lhr reduction of $K_{1}$ is accounted for in bounding skeletons.

Unfortunately, this connection does not always work. For instance, take:

$$
K_{2}=(\lambda x \cdot x *)(\lambda z \cdot(\lambda y \cdot y) z)
$$

When trying to represent $K_{2}$ as a hereditarily normal closure, we run into the issue that since $z$ is not a closed subterm, there is not way to replace the redex $(\lambda y . y) z$ by an environment. Of course, in $K_{1}$, we also had a generalized redex $(\lambda y, x)$ where $x$ is not closed. But in $K_{1}, x$ was active, in the sense that we had a redex $(\lambda x, \lambda z . z)$, so we knew how to define the environment on $x$. On the other hand, $z$ is passive in $K_{2}$ : there is no generalized redex $(\lambda z, N)$. In summary, the issue with $K_{2}$ is that there is a generalized redex $(\lambda y, z)$ where $z$ (obviously) contains a passive free variable $z$, and because of that $K_{2}$ cannot be directly represented as a hereditarily normal closure. 
Definition 3. A variable $x$ in $M$ is active iff it is a free variable or if there is a generalized redex $(\lambda x, N)$ in $M$. It is passive otherwise. A term $M$ is locally scoped (abbreviated l.s.) if for any generalized redex $(\lambda x, N)$ in $M$ all the free variables in $N$ are active in $M$. Likewise, $M$ is strongly locally scoped (abbreviated s.l.s.) if for any generalized redex $(\lambda x, N)$ in $M, N$ is closed.

So, the term $K_{1}$ above is locally scoped, but $K_{2}$ is not since there is a generalized redex $(\lambda y, z)$ with $z$ passive. Neither of those are strongly locally scoped. Any $\beta$-normal term is strongly locally scoped, and so is any term obtained by applications of $\beta$-normal forms (such as $\lambda$-terms corresponding to terms of combinatory logic). Local scope will be sufficient to ensure that the interpretation to bounding skeletons is a simulation, but the correspondence between terms and bounding skeletons will be tighter for strongly locally scoped terms: for those, the tree structure of the bounding skeleton will match the tree structure of imbricated generalized redexes. Strongly locally scoped terms are not stable under $\mathrm{lhr}$, so we need to develop the full connection on locally scoped terms instead.

Lemma 2. If $M$ is a locally scoped term of ground type and $M \rightarrow_{\mathrm{lhr}} M^{\prime}$, then $M^{\prime}$ is locally scoped.

\subsection{Interpretation in bounding skeletons}

Interpretation. The level of a type is defined by $\operatorname{lv}(o)=0$ and $\operatorname{lv}(A \rightarrow B)=$ $\max (\operatorname{lv}(A)+1, \operatorname{lv}(B))$. Likewise, the level $\operatorname{lv}(M)$ of a term $M$ is the level of its type. Finally, the order $\operatorname{ord}(M)$ of a term $M$ is the maximal $\operatorname{lv}(N)$, for all subterms $N$ of $M$. Within a term $\Gamma \vdash M: A$ such that $(x: B) \in \Gamma$, we write $\operatorname{lv}_{M}(x)=\operatorname{lv}(B)$. The term $M$ will generally be obvious from the context, so we will just write $\operatorname{lv}(x)$.

Definition 4. Let $\Gamma \vdash M: A$ be a term, with a bs-environment $\rho$, being defined as a partial function associating to each variable $x$ of $\Gamma$ on which it is defined a bounding skeleton $\rho(x)$. Then the bounding skeleton $\llbracket M \rrbracket \rho$ is defined by induction on the length of $M$, as follows:

$$
\begin{aligned}
& \llbracket * M_{1} \ldots M_{n} \rrbracket_{\rho}=0 \\
& \llbracket x_{0} M_{1} \ldots M_{n} \rrbracket_{\rho}=1+\bigsqcup_{i=1}^{n} \llbracket M_{i} \rrbracket_{\rho} \quad \text { if } \rho(x) \text { undefined } \\
& \llbracket x_{0} \quad M_{1} \ldots M_{n} \rrbracket_{\rho}=\left(1+\bigsqcup_{i=1}^{n} \llbracket M_{i} \rrbracket_{\rho}\right) \cdot \cdot_{\mathrm{lv}}(x)+1 \rho(x) \text { if } \rho(x) \text { defined } \\
& \llbracket \lambda x \cdot M \rrbracket_{\rho}=\llbracket M \rrbracket_{\rho} \\
& \llbracket(\lambda x . M) M_{1} \ldots M_{n} \rrbracket_{\rho}=\llbracket \begin{array}{llll}
M & M_{2} & \ldots & \left.M_{n} \rrbracket_{\rho \cup\left\{x \mapsto \llbracket M_{1} \rrbracket_{\rho}\right.}\right\}
\end{array}
\end{aligned}
$$

We write $\llbracket M \rrbracket$ for $\llbracket M \rrbracket_{\emptyset}$.

Measures on terms and their preservation. To estimate lhr on s.l.s. terms, we need to define measures on terms that reflect the geometry of the corresponding bounding skeletons. So instead of the height, we have two alternative quantities. 
The depth $\operatorname{depth}(M)$ of a term $M$ is defined by induction on the length of $M$ :

$$
\begin{aligned}
\operatorname{depth}\left(* M_{1} \ldots M_{n}\right) & =1 \\
\operatorname{depth}\left(x_{0} M_{1} \ldots M_{n}\right) & =\max _{1 \leq i \leq n} \operatorname{depth}\left(M_{i}\right) \\
\operatorname{depth}(\lambda x . M) & =\operatorname{depth}(M) \\
\operatorname{depth}\left((\lambda x . M) M_{1} \ldots M_{n}\right) & =\max \left(\operatorname{depth}\left(M M_{2} \ldots M_{n}\right), \operatorname{depth}\left(M_{1}\right)+1\right)
\end{aligned}
$$

Likewise, the local height $\operatorname{lh}(M)$ of a term $M$ is defined by:

$$
\begin{aligned}
\operatorname{lh}\left(* M_{1} \ldots M_{n}\right) & =0 \\
\operatorname{lh}\left(x_{0} M_{1} \ldots M_{n}\right) & =1+\max _{1 \leq i \leq n} \operatorname{lh}\left(M_{i}\right) \\
\operatorname{lh}(\lambda x \cdot M) & =\operatorname{lh}(M) \\
\operatorname{lh}\left((\lambda x . M) M_{1} \ldots M_{n}\right) & =\max \left(\operatorname{lh}\left(M M_{2} \ldots M_{n}\right), \operatorname{lh}\left(M_{1}\right)\right)
\end{aligned}
$$

Then, we have the following lemma:

Lemma 3. If $M$ is a strongly locally scoped term, then we have:

$$
\operatorname{depth}(\llbracket M \rrbracket) \leq \operatorname{depth}(M) \quad \max (\llbracket M \rrbracket) \leq \operatorname{lh}(M) \quad \operatorname{ord}(\llbracket M \rrbracket) \leq \operatorname{ord}(M)
$$

Simulation. In order to have our simulation result of linear head reduction into bounding skeletons, we need the additional requirement that the terms being interpreted are $\eta$-long - it is natural since our tools originate from game semantics, in which strategies are representations of $\eta$-long normal forms. As usual, $\eta$-expansion is the rule $M \rightarrow_{\eta} \lambda x^{A}$.M $x$, that applies when $M$ has type $A \rightarrow B$ and $x \notin \mathrm{fv}(M)$. Non $\beta$-normal $\eta$-long terms are often defined as the terms on which any further $\eta$-expansion creates a new $\beta$-redex. Since we have generalized the notion of redex, we instead define them as the terms for which any $\eta$-expansion creates a new generalized redex. Then, $\eta$-long terms are stable under lhr. Moreover, we have:

Proposition 1 (Simulation). Let $\Gamma \vdash M, M^{\prime}:$ o be $\eta$-long locally scoped terms, and suppose $M \rightarrow_{\mathrm{hr}} M^{\prime}$. Then, there is a such that $\llbracket M \rrbracket \rightarrow_{\mathrm{bs}} a \hookleftarrow \llbracket M^{\prime} \rrbracket$.

\subsection{Bounds for strongly locally scoped terms}

As a first application, we give exact bounds for the maximal length of lhr on strongly locally scoped terms. Formally, we will estimate the following quantity.

$\operatorname{Lls}_{n}(h, d)=\max \{\mathcal{N}(M) \mid \operatorname{ord}(M) \leq n \& \operatorname{lh}(M) \leq h \& \operatorname{depth}(M) \leq d \& M$ s.l.s. $\}$

To express our results, we will use some standard notations for comparing growth rates of functions. For functions $f, g: \mathbb{N} \rightarrow \mathbb{N}$, we write $f(n)=\Theta(g(n))$ 
when there exists reals $c_{1}, c_{2}>0$ and $N \in \mathbb{N}$ such that for all $n \geq N, c_{1} g(n) \leq$ $f(n) \leq c_{2} g(n)$. This is generalized to functions of multiple variables $f, g: \mathbb{N}^{p} \rightarrow \mathbb{N}$ by setting that $f\left(n_{1}, \ldots, n_{p}\right)=\Theta\left(g\left(n_{1}, \ldots, n_{p}\right)\right)$ iff there are $c_{1}, c_{2}>0$ and $N_{i} \in \mathbb{N}$ for all $i \in\{1, \ldots, p\}$ such that for all $n_{i} \geq N_{i}$ we have $c_{1} g\left(n_{1}, \ldots, n_{p}\right) \leq$ $f\left(n_{1}, \ldots, n_{p}\right) \leq c_{2} g\left(n_{1}, \ldots, n_{p}\right)$. If $h: \mathbb{N} \rightarrow \mathbb{N}$ is another function, we write $f\left(n_{1}, \ldots, n_{p}\right)=h\left(\Theta\left(g\left(n_{1}, \ldots, n_{p}\right)\right)\right)$ iff there is a function $\phi: \mathbb{N}^{p} \rightarrow \mathbb{N}$ such that $f\left(n_{1}, \ldots, n_{p}\right)=h\left(\phi\left(n_{1}, \ldots, n_{p}\right)\right)$ and $\phi\left(n_{1}, \ldots, n_{p}\right)=\Theta\left(g\left(n_{1}, \ldots, n_{p}\right)\right)$.

$\eta$-long form. Our simulation result only applies to $\eta$-long terms. Therefore, in order to obtain the upper bound we first need the following result:

Proposition 2. If $M$ is a term, then there is an $\eta$-long term $M^{\prime}$ such that:

$$
\begin{aligned}
\operatorname{lh}\left(M^{\prime}\right) & \leq \operatorname{lh}(M)+\operatorname{ord}(M) & \operatorname{depth}\left(M^{\prime}\right) & =\operatorname{depth}(M) \\
\operatorname{ord}\left(M^{\prime}\right) & =\operatorname{ord}(M) & \mathcal{N}\left(M^{\prime}\right) & \geq \mathcal{N}(M)
\end{aligned}
$$

Moreover if $M$ was s.l.s., $M^{\prime}$ is still s.l.s..

The proof is mostly direct, but rather long and technical. We show first that $\eta$-expansion can only increase the norm and that it preserves strong local scope and the order of terms. Moreover, if $\eta$-expansion is restricted so that it does not create new generalized redexes, then it terminates on an $\eta$-long form. Besides, restricted $\eta$-expansion preserves depth and a variant $\operatorname{lh}^{\prime}(M)$ of $\operatorname{lh}(M)$ such that $\operatorname{lh}(M) \leq \operatorname{lh}^{\prime}(M) \leq \operatorname{lh}(M)+\operatorname{ord}(M)$, taking into account the potential size of the variables that are not yet expanded. Details are omitted.

Upper bound. If $\Gamma \vdash M: A_{1} \rightarrow \ldots \rightarrow A_{n} \rightarrow o$ is a s.l.s. term, we first make it of ground type by forming $\Gamma \vdash M *_{A_{1}} \ldots *_{A_{n}}: o$ - its norm can only increase, the other quantities stay unchanged and the term is still s.l.s.. By Proposition 2, there is $M^{\prime} \eta$-long, of ground type, and s.l.s. such that $\operatorname{lh}\left(M^{\prime}\right) \leq \operatorname{lh}(M)+\operatorname{ord}(M)$, $\operatorname{depth}\left(M^{\prime}\right)=\operatorname{depth}(M), \operatorname{ord}\left(M^{\prime}\right)=\operatorname{ord}(M)$ and $\mathcal{N}\left(M^{\prime}\right) \geq \mathcal{N}(M)$. Along with Lemma 3, Proposition 1 and Theorem 1 this gives the following proposition.

Proposition 3. Suppose $M$ is a strongly locally scoped term of order at least one. Then, $\mathcal{N}(M) \leq 2_{\operatorname{ord}(M)-1}^{\operatorname{depth}(M) \log (\operatorname{lh}(M)+\operatorname{ord}(M)+1)}$.

Lower bound. We now set to prove the optimality of this upper bound by exhibiting a family of terms whose reduction length asymptotically reaches it. The family we describe is a variant of one used by Beckmann in [2], constructed by iterated exponentiation of Church numerals. We define higher types for Church integers by setting $A_{-2}=o$ and $A_{n+1}=A_{n} \rightarrow A_{n}$. Then, writing $\underline{n}_{p}$ for the Church integer for $n$ of type $A_{p}$, we define, for $n, k, p \geq 0$ and $M: A_{p}$ :

$$
[n]_{p}^{0}(M)=M \quad[n]_{p}^{k+1}(M)=\underline{n}_{p+1}[n]_{p}^{k}(M)
$$

One can immediately check that $[n]_{p}^{k}(M): A_{p}$ and that for all $q \in \mathbb{N},[n]_{p}^{k}\left(\underline{q}_{p}\right) \rightarrow_{\beta}^{*}$ ${\underline{q^{n^{k}}}}_{p}$. Exploiting this construction we set, for $n, k, p \geq 0$ :

$$
S_{n, k, p}=[n]_{p}^{k}\left(\underline{2}_{p}\right) \underline{2}_{p-1} \cdots \underline{2}_{0}
$$


For which it is immediate to check that for all $n, k, p \geq 0$ we have $S_{n, k, p} \rightarrow_{\beta}^{*}$ $2_{p}^{2^{n^{k}}}$. Moreover, by construction of $S_{n, k, p}$, for $n \geq 2$ and $p, k \geq 1$ we have

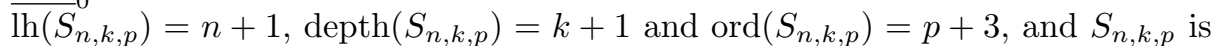
s.l.s.. To deduce a lower bound from this, we need to relate it to lhr using:

Lemma 4. If $M \rightarrow_{\beta}^{*} \underline{n}_{0}$, then $\mathcal{N}\left(M \mathrm{id}_{o}\right) \geq n$, where $\mathrm{id}_{o}=\lambda x^{o} \cdot x$.

Proof. By induction on $n$, exploiting that lhr preserves $\beta$-equivalence.

Theorem 2. For fixed $n \geq 2$ we have $\operatorname{Lls}_{n}(h, d)=2_{n-1}^{\Theta(d \log (h))}$.

Proof. Let us first consider the case where $n \geq 3$, as $n=2$ requires a separate construction for the lower bound. Let us fix $h \geq 3$ and $d \geq 2$. By Proposition 3, we already know that $\operatorname{Lls}_{n}(d, h) \leq 2_{n-1}^{d \log (h+n+1)}$. Moreover, we have $\operatorname{lh}\left(S_{h-1, d-1, n-3} \operatorname{id}_{o}\right)=h$ and $\operatorname{depth}\left(T_{h-1, d-1, n-3} \operatorname{id}_{o}\right)=d$, and by Lemma 4 we have $\mathcal{N}\left(T_{h-1, d-1, n-3} \operatorname{id}_{o}\right) \geq 2_{n-1}^{(d-1)} \log (h-1)$. To summarize:

$$
2_{n-1}^{(d-1) \log (h-1)} \leq \operatorname{Lls}_{n}(d, h) \leq 2_{n-1}^{d \log (h+n+1)}
$$

Therefore, with $n \geq 3$ fixed and $d, h$ parameters we have $\operatorname{Lls}_{n}(h, d)=2_{n-1}^{\Theta(d \log (h))}$.

For $n=2$, the upper bound still holds. For $d, p \geq 2$, define:

$$
U_{n, d}=\underline{n}_{1}\left(\underline{n}_{1} \ldots\left(\underline{n}_{1} \operatorname{id}_{o}\right) \ldots\right)
$$

where there are $d$ copies of $\underline{n}_{1}$ in total. Then, the term $U_{n, d}$ is s.l.s. and we have $\operatorname{lh}\left(U_{n, d}\right)=n+1, \operatorname{depth}\left(U_{n, d} \operatorname{id}_{o}\right)=d+1, \operatorname{ord}\left(U_{n, d} \operatorname{id}_{o}\right)=2$ and $\mathcal{N}\left(U_{n, d}\right) \geq$ $n^{d}=2^{d \log (n)}$. It follows that $\operatorname{Lls}_{2}(d, h)=2^{\Theta(d \log (h))}$.

In particular, reduction length for s.l.s. second-order terms of fixed depth is bounded by a polynomial of degree less than the depth.

\section{Exact bounds for general terms}

\subsection{Lambda-lifting to strongly locally scoped terms}

In order to deduce bounds for general terms, we now describe a transformation taking any $\lambda$-term $M$ to a corresponding s.l.s. term $M^{\prime}$; this transformation is a variant of the familiar notion of $\lambda$-lifting [12], adapted to lift variables through generalized redexes as well as $\beta$-redexes.

Take a term $M=\lambda x^{A} .\left(\lambda y^{A} . y\right) x$. Obviously, $M$ is not s.l.s.: indeed there is a prime redex $(\lambda y, x)$ and the subterm $x$ has $x$ free. In order to make the variable $x$ "local", we modify the abstraction subterm $\lambda y . y$ to forward explicitly the variable $x$. We get the term $M^{\prime}=\lambda x^{A} \cdot\left(\lambda y^{A \rightarrow A} \cdot y x\right)\left(\lambda x^{\prime A} \cdot x^{\prime}\right)$. The type of $y$ has changed, but not the type of the overall term. Note that the terms $M$ and $M^{\prime}$ are still $\beta$-equivalent, although we are not going to use that explicitly. More importantly, the norm has increased, the order has increased by one, and 


$$
\begin{gathered}
\frac{y \in \mathrm{fv}\left(M_{1}\right)}{(\lambda x . M) M_{1} \ldots M_{n} \rightarrow_{\lambda 1}(\lambda x . M[x y / x])\left(\lambda y^{\prime} . M_{1}\left[y^{\prime} / y\right]\right) \ldots M_{n}} \\
\frac{M_{i} \rightarrow_{\lambda 1} M_{i}^{\prime}}{x_{0} M_{1} \ldots M_{n} \rightarrow_{\lambda 1} x_{0} M_{1} \ldots M_{i}^{\prime} \ldots M_{n}} \\
\frac{M_{1} \rightarrow_{\lambda 1} M_{1}^{\prime}}{\lambda x . M \rightarrow_{\lambda 1} \lambda x \cdot M^{\prime}} \\
\frac{(\lambda x . M) M_{1} \ldots M_{n} \rightarrow_{\lambda 1}(\lambda x \cdot M) M_{1}^{\prime} \ldots M_{n}}{(\lambda x . M) M_{1} \ldots M_{n} \rightarrow_{\lambda 1}\left(\lambda x \cdot M^{\prime}\right) M_{1} M_{2}^{\prime} \ldots M_{n}^{\prime}}
\end{gathered}
$$

Fig. 3. Definition of the $\lambda$-lifting expansion $\rightarrow_{\lambda 1}$

the other quantities are essentially unchanged. We formalize this construction by the $\lambda$-lifting expansion $\rightarrow_{\lambda 1}$, defined in Figure 3.

In general $\rightarrow_{\lambda 1}$ leaves the type unchanged, although it can change the type of bound variables. Moreover $\rightarrow_{\lambda 1}$ terminates, and its normal form is necessarily s.l.s.. Altogether, we have the following result:

Lemma 5. For any term $M$, there is a strongly locally scoped $M^{\prime}$ such that:

$$
\begin{array}{rlrl}
\operatorname{lh}\left(M^{\prime}\right) & \leq \operatorname{lh}(M)+1 & \operatorname{depth}\left(M^{\prime}\right) & =\operatorname{depth}(M) \\
\operatorname{ord}\left(M^{\prime}\right) & \leq \operatorname{ord}(M)+1 & \mathcal{N}\left(M^{\prime}\right) & \geq \mathcal{N}(M)
\end{array}
$$

This is established by a rather lengthy technical proof, studying commutations between $\rightarrow_{\lambda 1}$ and $\rightarrow_{\mathrm{lhr}}$. Preservation of depth is easy since we do not add generalized redexes, and (relative) preservation of order and local height is established as for $\rightarrow_{\eta}$, by building variants $\mathrm{lh}^{\prime}$ and ord' which take into account the potential expansion of variables, that satisfy $\operatorname{lh}(M) \leq \operatorname{lh}^{\prime}(M) \leq \operatorname{lh}(M)+1$ and $\operatorname{ord}(M) \leq \operatorname{ord}^{\prime}(M) \leq \operatorname{ord}(M)+1$ and are preserved by $\rightarrow_{\lambda 1}$.

\subsection{Expanding variables}

For non locally scoped terms, the local height and the depth are rather unnatural quantities, and the bounds are not naturally expressed in terms of them. We convert one to the other using another norm-increasing term transformation.

Lemma 6. For any term $M$, there exists a term $M^{\prime}$ such that $M \rightarrow_{\eta^{*}} M^{\prime}$,

$$
\begin{aligned}
\operatorname{lh}\left(M^{\prime}\right) & \leq 2 & \operatorname{depth}\left(M^{\prime}\right) & \leq \mathrm{h}(M) \\
\operatorname{ord}\left(M^{\prime}\right) & =\operatorname{ord}(M) & \mathcal{N}\left(M^{\prime}\right) & \geq \mathcal{N}(M)
\end{aligned}
$$


The term $M^{\prime}$ is obtained by replacing each occurrence $x_{0}$ in $M$ of a variable $x: A_{1} \rightarrow \ldots \rightarrow A_{n} \rightarrow o$ by its $\eta$-expanded form $\lambda y^{1^{A_{1}}} \ldots \lambda y^{n A_{n}} \cdot x_{0} y^{1} \ldots y^{n}$. Since we have $M \rightarrow_{\eta^{*}} M^{\prime}$, we already know that $\mathcal{N}\left(M^{\prime}\right) \geq \mathcal{N}(M)$, the other inequalities are easily established by induction.

\subsection{Exact bounds for general terms}

In this section, we are interested in estimating the quantity:

$$
\operatorname{Lgen}_{n}(h)=\max \{\mathcal{N}(M) \mid \operatorname{ord}(M) \leq n \& \mathrm{~h}(M) \leq h\}
$$

We do that by applying the tools developed earlier to get an upper bound on the length of reduction, and then prove a matching lower bound by providing terms whose length of reduction asymptotically reaches the upper bound.

Upper bound. Starting from a term $M$, we first expand variables using Lemma 6, then make it s.l.s. using Lemma 5. This gives $M^{\prime}$ such that:

$$
\begin{array}{rlrl}
\operatorname{lh}\left(M^{\prime}\right) & \leq 3 & \operatorname{depth}\left(M^{\prime}\right) & =\mathrm{h}(M) \\
\operatorname{ord}\left(M^{\prime}\right) & \leq \operatorname{ord}(M)+1 & \mathcal{N}\left(M^{\prime}\right) & \geq \mathcal{N}(M)
\end{array}
$$

By applying Proposition 3, we get:

Proposition 4. Suppose $M$ is a term. Then, $\mathcal{N}(M) \leq 2_{\operatorname{ord}(M)}^{\mathrm{h}(M) \log (\operatorname{ord}(M)+5)}$.

Lower bound. We provide a lower bound matching asymptotically the upper bound offered by Proposition 4 . The construction is essentially the same as the one used in [2] for the lower bound in terms of height.

For $p \geq 1$ and $k \geq 0$, we define $b_{0}^{p}=\underline{2}_{p}$ and $b_{k+1}^{p}=\lambda x^{A_{p-1}} \cdot b_{k}^{p}\left(b_{k}^{p} x\right)$. Then, we set:

$$
B_{k}^{p}=b_{k}^{p} \underline{2}_{p-1} \cdots \underline{2}_{0}
$$

Note that this term is not s.l.s.. By standard arithmetic of Church numerals, we have that for any $p \geq 1, k \geq 0, B_{k}^{p} \rightarrow_{\beta}^{*} 2_{p+2_{0}}^{k}$. By Lemma 4 it follows that $\mathcal{N}\left(B_{k}^{p} \operatorname{id}_{o}\right) \geq 2_{p+2}^{k}$. It is direct to check that $\operatorname{ord}\left(B_{k}^{p}\right)=p+2$ and $\mathrm{h}\left(B_{k}^{p}\right)=k+3$ (for $k \geq 1$ ). Therefore, we have:

Theorem 3. For fixed $n \geq 3$ we have $\operatorname{Lgen}_{n}(h)=2_{n}^{\Theta(h)}$.

For a term $M$ of height $h$ and order $n$, Beckmann's results [2] predict that any $\beta$-reduction chain of $M$ terminates in less than $2_{n+1}^{\Theta(h)}$ steps. It might seem counter-intuitive that our bound (with linear head reduction) is smaller than Beckmann's (with $\beta$-reduction) since we substitute only one occurrence at a time, which is obviously longer. However, Beckmann considers arbitrary $\beta$-reduction, not head $\beta$-reduction. The possibility of reducing in arbitrary locations of the term unlocks much longer reductions, since higher-order free variables or constants can isolate sections of the term that will never arrive in head position but can still be affected by arbitrary $\beta$-reduction. The fact that the length of linear head reduction has the same order of magnitude as head $\beta$-reduction is not surprising in the light of Accattoli and Dal Lago's recent result [1] that a similar notion of linear head reduction is quadratically related to head reduction. 


\section{Conclusion}

We have worked out the precise connection between bounding skeletons and syntactic reduction, deducing bounds for linear head reduction in the simplytyped $\lambda$-calculus. The analysis uncovers locally scoped terms, whose reduction relates closely to game-theoretic interaction. Through this work, we obtain syntaxindependent tools to reason on the complexity of programs, hopefully useful in implicit complexity. Although we have only described this connection here for the pure $\lambda$-calculus, the connection with games suggest that similar constructions should yield the same results for languages with effects such as control, nondeterminism or ground state. In future work we plan plan to generalize these tools to more expressive languages, in particular in the presence of recursion.

Acknowledgment. We gratefully acknowledge the support of the ERC Advanced Grant ECSYM.

\section{References}

1. Beniamino Accattoli and Ugo Dal Lago. On the invariance of the unitary cost model for head reduction. In Ashish Tiwari, editor, RTA, volume 15 of LIPIcs, pages 22-37. Schloss Dagstuhl - Leibniz-Zentrum fuer Informatik, 2012.

2. Arnold Beckmann. Exact bounds for lengths of reductions in typed lambdacalculus. J. Symb. Log., 66(3):1277-1285, 2001.

3. Alexis Bernadet and Stéphane Lengrand. Complexity of strongly normalising terms via non-idempotent intersection types. In FOSSACS, pages 88-107, 2011.

4. Pierre Clairambault. Estimation of the length of interactions in arena game semantics. In FOSSACS, pages 335-349, 2011.

5. Thierry Coquand. A semantics of evidence for classical arithmetic. J. Symb. Log., 60(1):325-337, 1995.

6. V. Danos, H. Herbelin, and L. Regnier. Game semantics and abstract machines. In Logic in Computer Science, 1996. LICS'96. Proceedings., Eleventh Annual IEEE Symposium on, pages 394-405. IEEE, 1996.

7. V. Danos and L. Regnier. How abstract machines implement head linear reduction. 2003. unpublished.

8. Vincent Danos and Jean-Baptiste Joinet. Linear logic and elementary time. Inf. Comput., 183(1):123-137, 2003.

9. Daniel de Carvalho, Michele Pagani, and Lorenzo Tortora de Falco. A semantic measure of the execution time in linear logic. TCS, 412(20):1884-1902, 2011.

10. Jean-Yves Girard. Light linear logic. In Daniel Leivant, editor, $L C C$, volume 960 of Lecture Notes in Computer Science, pages 145-176. Springer, 1994.

11. J. M. E. Hyland and C.-H. Luke Ong. On full abstraction for PCF: I, II, and III. Inf. Comput., 163(2):285-408, 2000.

12. Thomas Johnsson. Lambda lifting: Transforming programs to recursive equations. In FPCA, pages 190-203, 1985.

13. Ugo Dal Lago and Olivier Laurent. Quantitative game semantics for linear logic. In Michael Kaminski and Simone Martini, editors, CSL, volume 5213 of Lecture Notes in Computer Science, pages 230-245. Springer, 2008.

14. H. Schwichtenberg. An upper bound for reduction sequences in the typed $\lambda$ calculus. Archive for Mathematical Logic, 30(5):405-408, 1991. 